\title{
Clinical efficacy of aspirin combination treatment in the treatment of coronary heart disease and its effect on inflammatory factors: a systematic review and meta-analysis
}

\author{
Ping Mao, Xiaowei Liu, Yingzheng Weng, Lijiang Tang, Yimin Tang \\ Department of Cardiovascular Diseases, Zhejiang Hospital, Hangzhou, China \\ Contributions: (I) Conception and design: P Mao, Y Tang; (II) Administrative support: Y Tang; (III) Provision of study materials or patients: L Tang, F \\ Lv, C Xu; (IV) Collection and assembly of data: P Mao, D Lu, Y Tang; (V) Data analysis and interpretation: P Mao, Y Tang; (VI) Manuscript writing: \\ All authors; (VII) Final approval of manuscript: All authors. \\ Correspondence to: Yimin Tang. Department of Cardiovascular Diseases, Zhejiang Hospital, Hangzhou 310013, China. Email: Dr_TangYM@163.com.
}

\begin{abstract}
Background As the mainstay treatment for coronary heart disease (CHD), aspirin alone is reported to be less effective than in combination when treating CHD. The aim of this analysis was to systematically evaluate the efficacy and safety of aspirin in combination with other drugs for the treatment of CHD, as well as its effect on the levels of inflammatory factors.
\end{abstract}

Methods: Electronic databases were searched from 2011 to 2021 and randomized controlled trials (RCTs) on aspirin in CHD patients were included in our study. Data was statistically analyzed using Stata 16.0 (StataCorp).

Results: A total of 13 RCTs were included, with a total of 1,442 patients. Compared with control group (aspirin alone) group, the response rate in the treatment group (aspirin in combination with other drugs) was significantly improved [odds ratio $(\mathrm{OR})=5.11 ; 95 \%$ confidence interval $(\mathrm{CI}): 3.56-7.35$ ], while the incidence of adverse reactions was markedly decreased (OR $=0.36$; 95\% CI: $0.25-0.53)$. Before treatment, no significant differences were identified in the levels of inflammatory factors between the groups The inflammatory factors included C-reactive protein (CRP), interleukin-6 (IL-6), and tumor necrosis factor- $\alpha$ (TNF- $\alpha$ ). After treatment, CRP and TNF- $\alpha$ levels were significantly lower in both groups compared with those before treatment. However, there was no statistically significant difference in IL-6 levels after treatment between the groups.

Discussion: Aspirin is effective in the treatment of CHD, both alone and in combination. However, the latter has higher clinical efficacy and safety, and can significantly reduce the level of inflammatory factors in CHD patients.

Keywords: Aspirin; coronary heart disease (CHD); clinical effect; inflammatory factors; meta-analysis

Submitted May 27, 2021. Accepted for publication Jul 26, 2021.

doi: 10.21037/apm-21-1648

View this article at: https://dx.doi.org/10.21037/apm-21-1648

\section{Introduction}

Coronary heart disease (CHD), also known as coronary artery disease, refers to a cardiovascular disease caused by atherosclerosis of the coronary arteries that results in vascular stenosis and obstruction and subsequent myocardial ischemia and necrosis (1). The incidence of CHD continues to rise. In 2017, this disease became the leading cause of death in the global population, resulting in about 8.92 million deaths and a standardized mortality ratio of 142/100,000. In China, CHD-caused deaths increased from $8.6 \%$ in 1990 to $15.2 \%$ in 2013 (2).

CHD results from a combination of factors which include smoking, alcohol consumption, unhealthy eating habits, and insufficient physical activity $(3,4)$. CHD is a 
chronic inflammatory disease with inflammation present through all stages of atherosclerosis, and inflammation may be a common link or pathway in the pathogenic mechanism of multiple atherogenic factors (5). The American College of Cardiology/American Heart Association (ACC/AHA) Guidelines for Primary Prevention of Cardiovascular Disease propose that the principles of primary prevention of CHD can be summarized as aspirin, blood pressure stabilization, cholesterol control, and smoking cessation (ABCS) (6). Aspirin has been clinically shown to have effects on reducing the expression of inflammatory cytokines, antiatherosclerosis, antiplatelet, and preventing thrombosis (7). Specifically, this drug reduces platelet aggregation by irreversible acetylation of cyclooxygenase-1 (COX-1), thereby inhibiting the conversion of arachidonic acid (AA) to thromboxane A2 (TXA A2) $(8,9)$. For patients with chronic CHD or peripheral artery disease (PAD), $2.5 \mathrm{mg}$ twice daily of rivaroxaban or $100 \mathrm{mg}$ daily of aspirin has been shown to reduce the incidence of cardiovascular disease-caused death, stroke, or myocardial infarction by $24 \%$, while increasing the occurrence of adverse effects, such as hemorrhage (10-12).

There is no consensus on the use of aspirin alone or in combination to prevent CHD, and there is still a lack of reliable evidence on the clinical efficacy and safety of aspirin in combination. With the aim of better guiding clinical practice, this meta-analysis compared the efficacy and safety of aspirin alone and in combination for the treatment of CHD, and examined its effect on the expression of inflammatory factors.

We present the following article in accordance with the PRISMA reporting checklist (available at https://dx.doi. org/10.21037/apm-21-1648).

\section{Methods}

\section{Search strategy}

Medical literature analysis and retrieval system (MEDLINE) was widely searched using the following online databases: PubMed, Web of Science, EMBASE, CNKI China, and Wanfang. The search time was set from 2011 to 2021, and the keywords were (I) "aspirin", (II) "coronary heart disease", (III) "inflammatory factors", and (IV) "clinical effect". The language was limited to English and Chinese.

\section{Inclusion and exclusion criteria}

The inclusion criteria were the following: (I) study subjects were CHD patients treated with aspirin; (II) intervention measures involved treatment group patients being treated with aspirin combined with clopidogrel/atorvastatin/ betaloc with the control group being given aspirin alone; (III) outcome measures included response rate, incidence of adverse reactions, C-reactive protein (CRP) before and after treatment, interleukin-6 (IL-6) before and after treatment, and tumor necrosis factor- $\alpha(\mathrm{TNF}-\alpha)$ before and after treatment.

Meanwhile, the exclusion criteria were as follows: (I) lack of comparison of treatment results in CHD patients treated with aspirin alone; (II) case reports, systematic reviews, and animal experiments; (III) literature without the data required by this meta-analysis or literature for which the original text could not be obtained; (IV) literature with poor quality or missing data; and (V) duplicate literature.

\section{Data extraction and quality assessment}

Two investigators independently extracted the data, including first author, year of publication, sample size, length of time patients were enrolled in the study, study type, endpoint event, and baseline characteristics. Any disagreement between the investigators was resolved through discussion with a third investigator.

\section{Statistical analysis}

Stata 16.0 (StataCorp) was the statistical tool used for data analysis. We calculated pooled odds ratios (ORs) for dichotomous endpoints with $95 \%$ confidence intervals (CIs). In case of heterogeneity $\left(\mathrm{P}>0.05\right.$ or $\left.\mathrm{I}^{2}<50 \%\right)$, the fixed-effects model (Mantel-Haenszel) was used; otherwise $\left(\mathrm{P}<0.05\right.$ or $\left.\mathrm{I}^{2}>50 \%\right)$, the random-effects model (M-Hheterogeneity) was adopted. Publication bias was assessed by funnel plots and Begg and Egger tests. A significant difference was suggested if $\mathrm{P}<0.05$. Sensitivity analysis was performed by sequentially excluding each study and observing whether the results obtained were significantly different.

\section{Results}

\section{Literature search results}

The specific flow chart of the literature screening process is shown in Figure 1. In total, 238 articles were retrieved from the databases, and 59 duplicates and 48 potentially relevant articles were excluded initially. After reading the titles and 

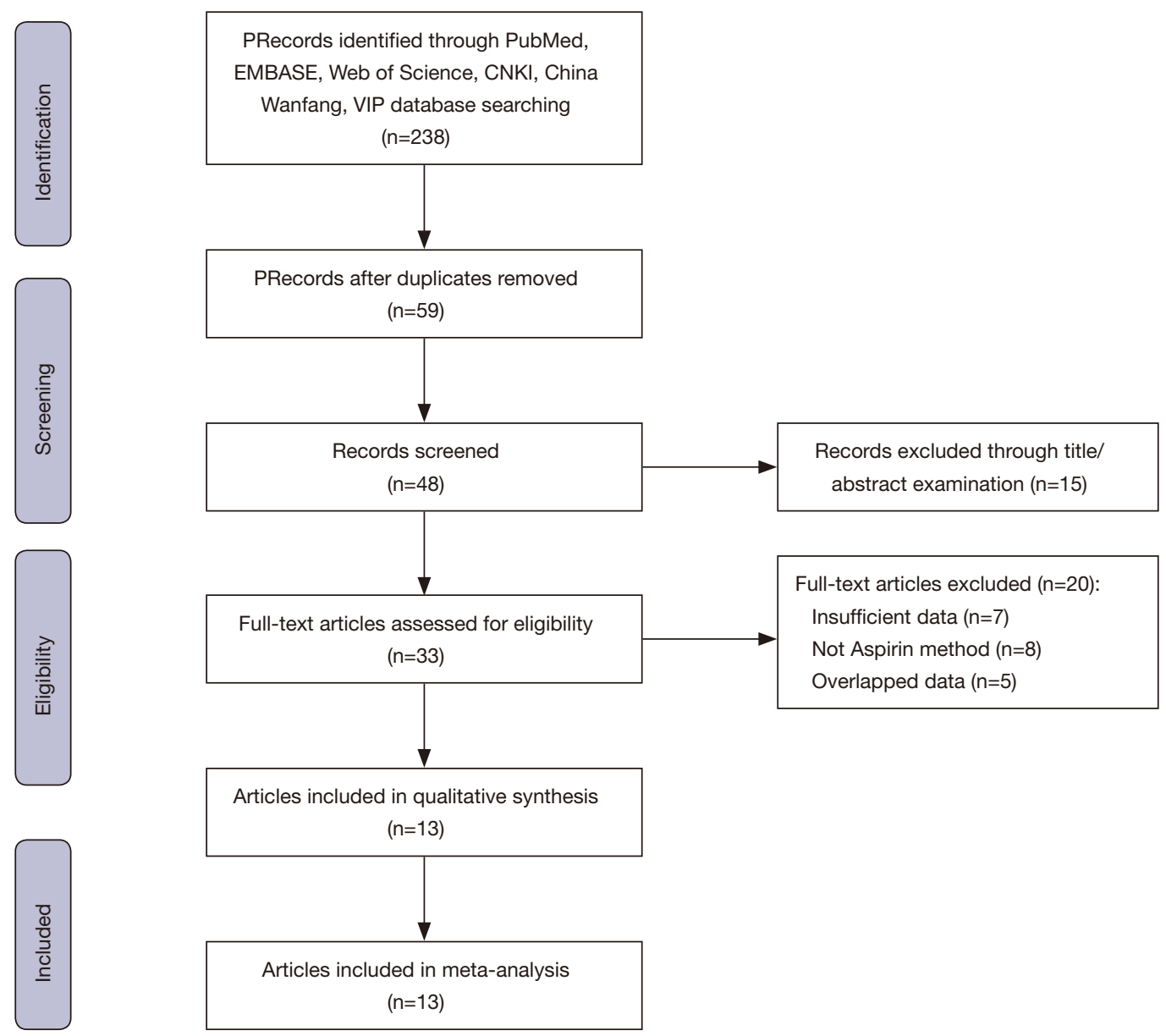

Figure 1 Flow chart of literature screening.

abstracts, 15 articles were excluded, and another 20 studies were excluded after the full text was read. Ultimately, 13 articles were included (13-25), with a total of 1,442 patients (treatment group: $\mathrm{n}=721$, control group: $\mathrm{n}=721$ ). All 13 studies were randomized controlled trials (RCTs), with sample sizes ranging from 64 to 256 patients. The average age of the patients ranged from 50 to 70 years, the male to female ratio was balanced, and the average follow-up time ranged from 1 year to 3 years. Baseline characteristics of the patients are shown in Table 1 .

\section{Primary endpoints}

\section{Clinical efficacy indicators}

Twelve studies reported response rate as the primary endpoint in both groups. No marked heterogeneity was present $\left(I^{2}=0.0 \% ; P=0.993\right)$. Incidence of adverse reactions was reported in 11 articles. No significant heterogeneity existed in the incidence of adverse reactions $\left(\mathrm{I}^{2}=0.0 \%\right.$; $\mathrm{P}=0.472)$. The fixed-effects model was used to evaluate the results of treatment effectiveness and incidence of adverse reactions. In comparison with the control group, the treatment group showed a significantly improved response rate $(\mathrm{OR}=5.11 ; 95 \% \mathrm{CI}: 3.56-7.35 ; \mathrm{P}<0.001$; Figure $2 A)$ and had a lower incidence of adverse reactions $(\mathrm{OR}=0.36$; 95\% CI: 0.24-0.53; $\mathrm{P}<0.001$; Figure $2 B$ ).

Publication bias was estimated through funnel plots. The funnel plot (Figure $3 A$ ) shows the publication bias of treatment effectiveness. A symmetric distribution of scatters indicating no effect of publication bias on the analysis results, which was confirmed by the Begg test $(\mathrm{Z}=0.21$; $\mathrm{P}=0.837>0.05)$ and Egger test $(\mathrm{t}=-0.01 ; \mathrm{P}=0.994>0.05)$. The funnel plot (Figure $3 B$ ) shows the publication bias of the incidence of adverse reactions was estimated. A symmetrical distribution of scatters indicating a small possibility of publication bias in the incidence of adverse reactions. This 


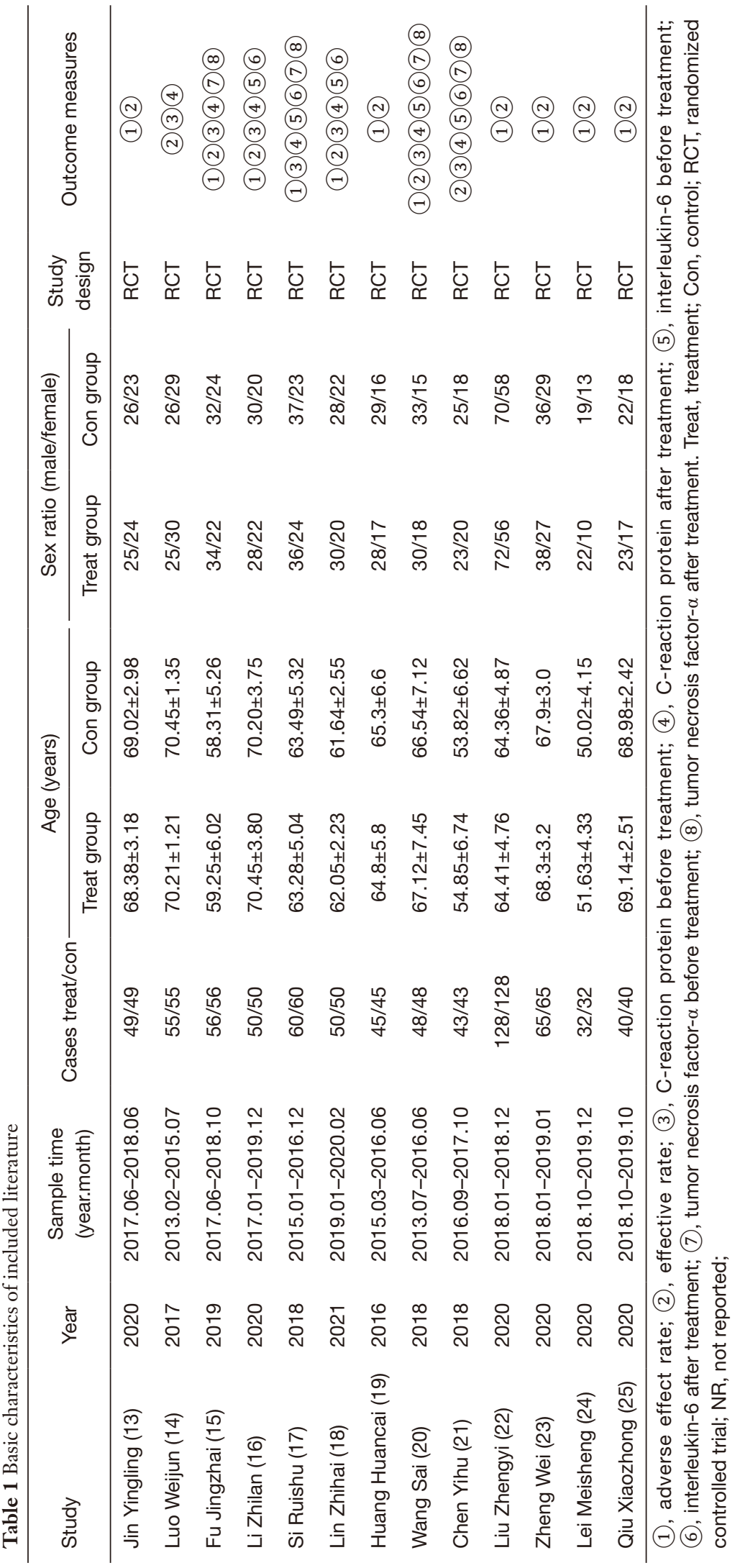




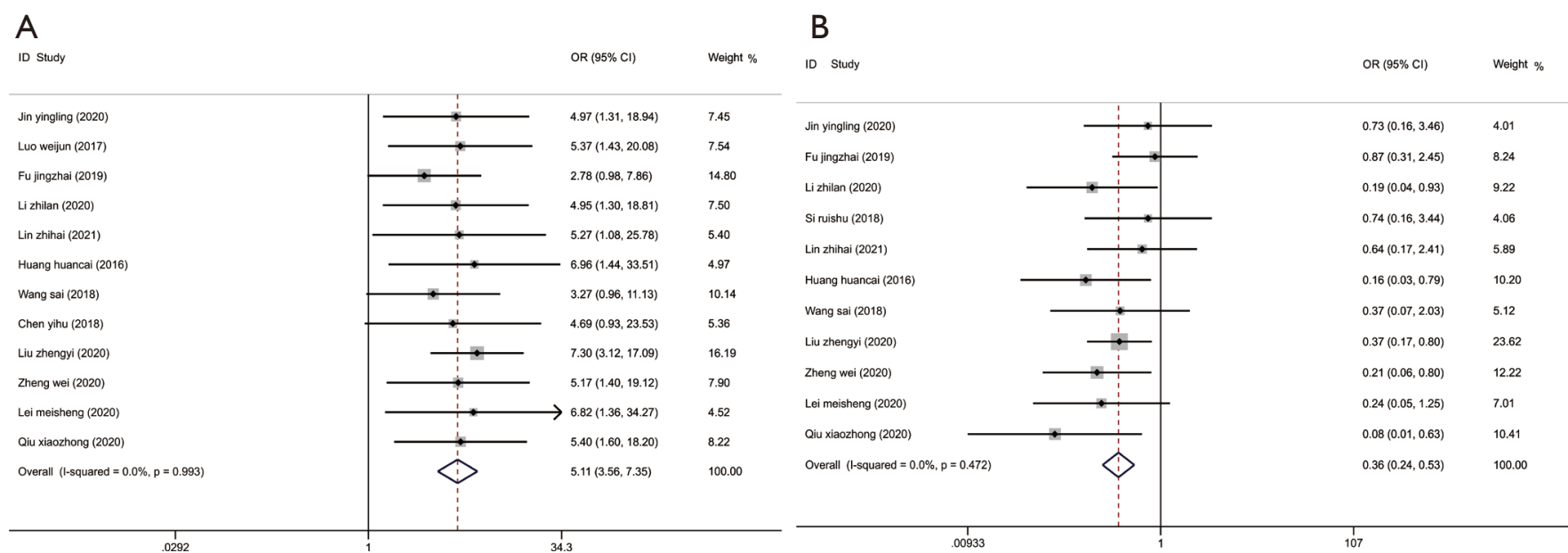

Figure 2 Forest plots of response rate (A) and incidence of adverse reactions (B) in the two groups. OR, odds ratio; CI, confidence interval.
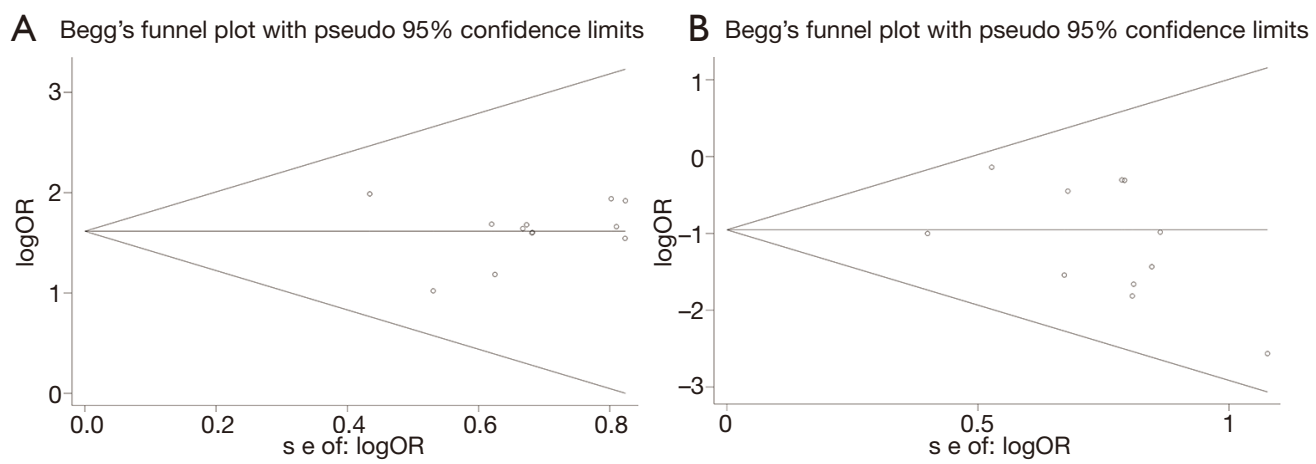

Figure 3 Funnel plots of response rate (A) and incidence of adverse reactions (B) in the two groups. OR, odds ratio.

was confirmed by the Begg test $(\mathrm{Z}=01.25 ; \mathrm{P}=0.213>0.05)$ and Egger test $(\mathrm{t}=-1.26 ; \mathrm{P}=0.241>0.05)$.

\section{Inflammatory factor levels before treatment}

Pretreatment inflammatory factors included CRP, IL-6, and TNF- $\alpha$. In all, 7 studies compared CRP levels between the 2 groups [standardized mean difference $(\mathrm{SMD})=0.20$; 95\% CI: -0.13 to $0.54 ; \mathrm{P}=0.234$; Figure $4 A$ ], 5 compared IL-6 levels (SMD $=-0.00,95 \%$ CI: -0.18 to $0.17 ; \mathrm{P}=0.969$; Figure $4 B$ ), and 4 compared TNF- $\alpha$ levels (SMD $=0.10$; 95\% CI: -0.09 to 0.29; $\mathrm{P}=0.317$; Figure $4 C$ ). Collectively, no significant difference was identified in inflammatory factor expression levels before treatment between the 2 groups. Therefore, the studies mentioned above were comparable. Because the number of included articles was less than 10 , no publication bias analysis was performed.

\section{Inflammatory factor levels after treatment}

The literature mentioning pretreatment inflammatory factors also reported the inflammatory factor levels after treatment. Due to the significant heterogeneity of CRP $\left(\mathrm{I}^{2}=97.7 \% ; \mathrm{P}<0.001\right)$, IL-6 $\left(\mathrm{I}^{2}=97.2 \% ; \mathrm{P}<0.001\right)$, and TNF- $\alpha$ levels $\left(\mathrm{I}^{2}=97.9 \% ; \mathrm{P}<0.001\right)$ after treatment, the random-effects models were used for analysis. As shown in Figure 5, in comparison with the control group, CRP expression (SMD $=-3.05$; 95\% CI: -4.27 to $-1.84 ; \mathrm{P}<0.001$,) and TNF- $\alpha$ expression (SMD $=-2.65,95 \%$ CI: -4.49 to $-0.81 ; \mathrm{P}=0.005)$ after treatment were lower in the treatment group. However, there was no significant difference in IL-6 expression after treatment between the 2 groups (SMD $=-0.99 ; 95 \%$ CI: -2.16 to $0.19 ; \mathrm{P}=0.101 ;$ Figure $5 B$ ).

Because the number of included articles was less than 10 , no publication bias analysis was performed. 
A
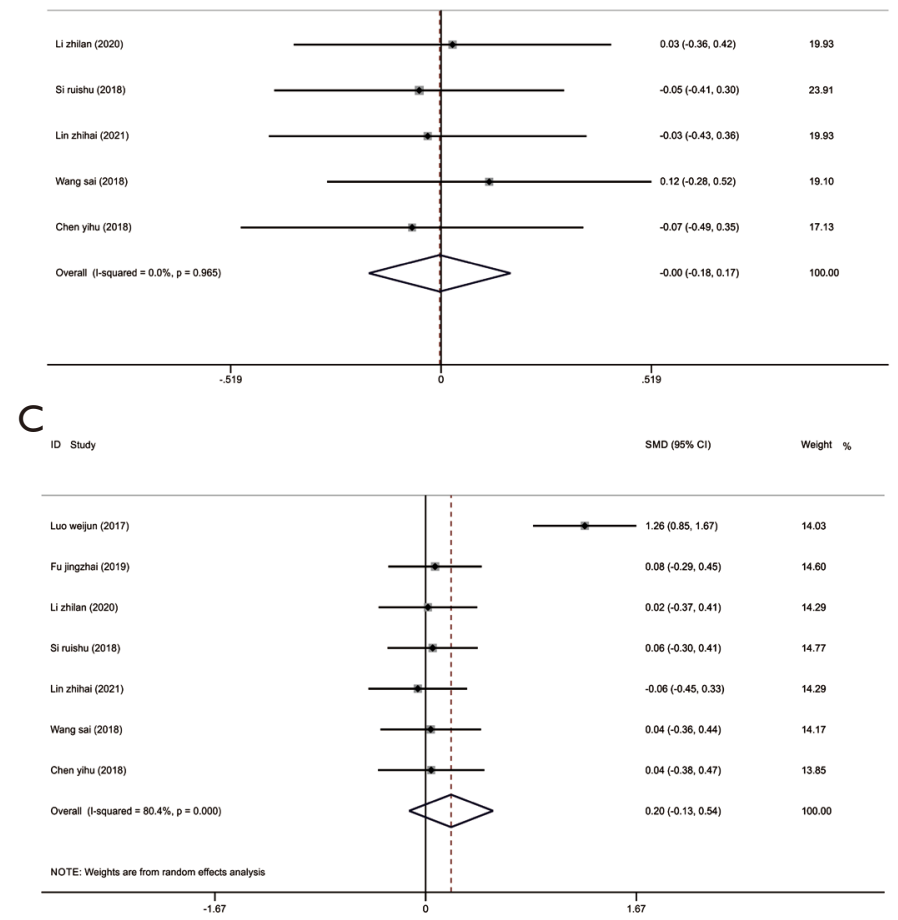

B

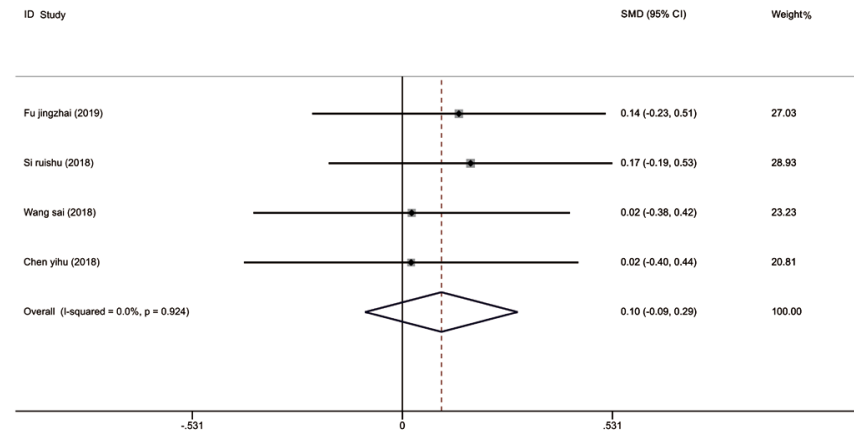

Figure 4 Forest plots of C-reactive protein (A), interleukin-6 (B), and tumor necrosis factor- $\alpha$ (C) levels before treatment. CI, confidence interval.

\section{Sensitivity analysis}

The included studies were removed one by one and the statistical model was changed for sensitivity analysis. The results showed no significant changes in the pooled effect size and CI for response rate (Figure $6 A$ ) and adverse reactions (Figure 6B), as well as for CRP level (Figure 7A) and IL-6 level (Figure $7 B$ ) before treatment, suggesting that this meta-analysis had good stability. However, the pooled effect size of TNF- $\alpha$ level (Figure $7 C$ ) changed greatly after removing the first article, indicating the possibility of heterogeneity. Additionally, after treatment, the pooled effect size and CI of CRP level (Figure 8A), IL-6 level (Figure $8 B$ ), and TNF- $\alpha$ level (Figure $8 C$ ) fluctuated, indicating that this meta-analysis was reliable.

\section{Discussion}

Clinically, lifelong administration of aspirin has good efficacy in reducing cardiovascular disease-related death, myocardial infarction, and stroke (26). Aspirin, as a conventional antiplatelet aggregation and antithrombotic drug, has been widely applied in the clinical treatment of CHD. However, aspirin alone has many more side effects, such as gastrointestinal hemorrhage and renal toxicity (2). Relevant systematic reviews have shown that aspirin increases the risk of massive hemorrhage by $54 \%$ (27). Whether aspirin in combination with other drugs can effectively treat CHD is still controversial, and whether its benefits outweigh its risks needs to be further explored. Hence, this meta-analysis specifically assessed the clinical efficacy and safety of aspirin in combination with other drugs, as well as the effect on inflammatory factor levels.

The 12 studies included in our meta-analysis reported the response rate in both groups. In comparison with the control group, the treatment group had a markedly improved response rate $(\mathrm{OR}=5.11 ; 95 \% \mathrm{CI}$ : 3.56-7.35). The incidence of adverse reactions was analyzed in 11 articles. In comparison with the control group, the incidence of adverse reactions was lower in the treatment group $(\mathrm{OR}=0.36$; 95\% CI: $0.25-0.53)$. Overall, the combination therapy of aspirin with other drugs significantly improved 


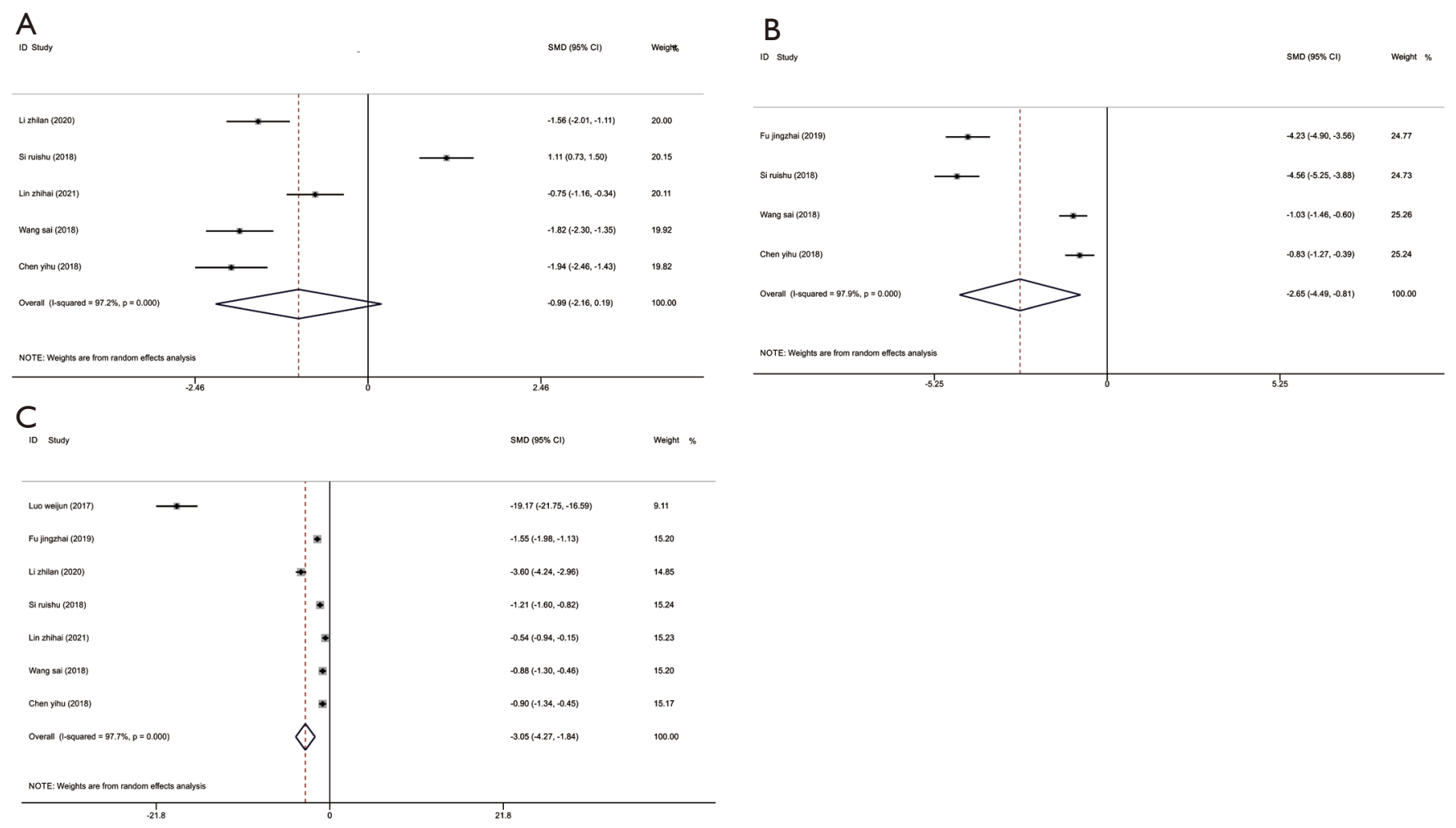

Figure 5 Forest plots of C-reactive protein (A), interleukin-6 (B), and tumor necrosis factor- $\alpha$ (C) levels after treatment. CI, confidence interval.
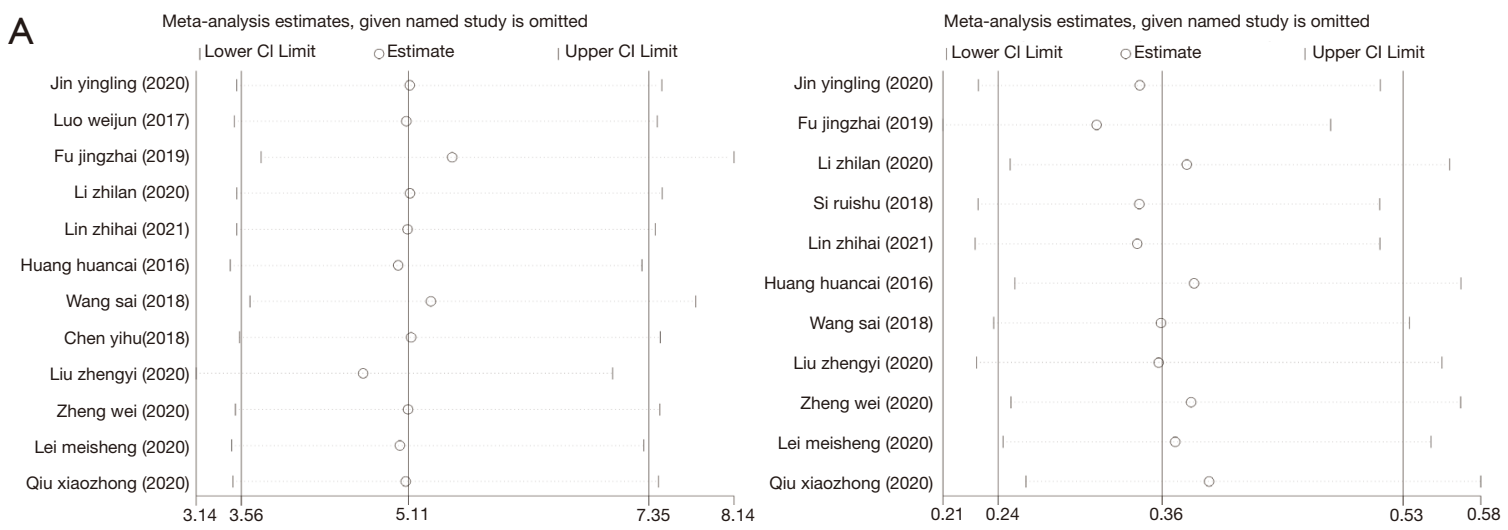

Figure 6 Sensitivity analysis of response rate (A) and incidence of adverse reactions (B) in the two groups. CI, confidence interval.

the treatment efficacy and reduced the possibility of adverse reactions, indicating that it has higher efficacy and safety in clinical use.

In terms of inflammatory factor levels, CRP expression $(\mathrm{SMD}=-3.05,95 \% \mathrm{CI}:-4.27$ to -1.84$)$ and TNF- $\alpha$ expression ( $\mathrm{SMD}=-2.65,95 \% \mathrm{CI}:-4.49$ to $-0.81 ; \mathrm{P}=0.005$ ) in the treatment group were significantly lower than those in the control group. However, no marked difference in IL-6 expression after treatment was identified between the 2 groups (SMD =-0.99; 95\% CI: -2.16 to 0.19). After treatment, both the CRP and TNF- $\alpha$ levels in the 2 groups were improved compared with those before treatment. 

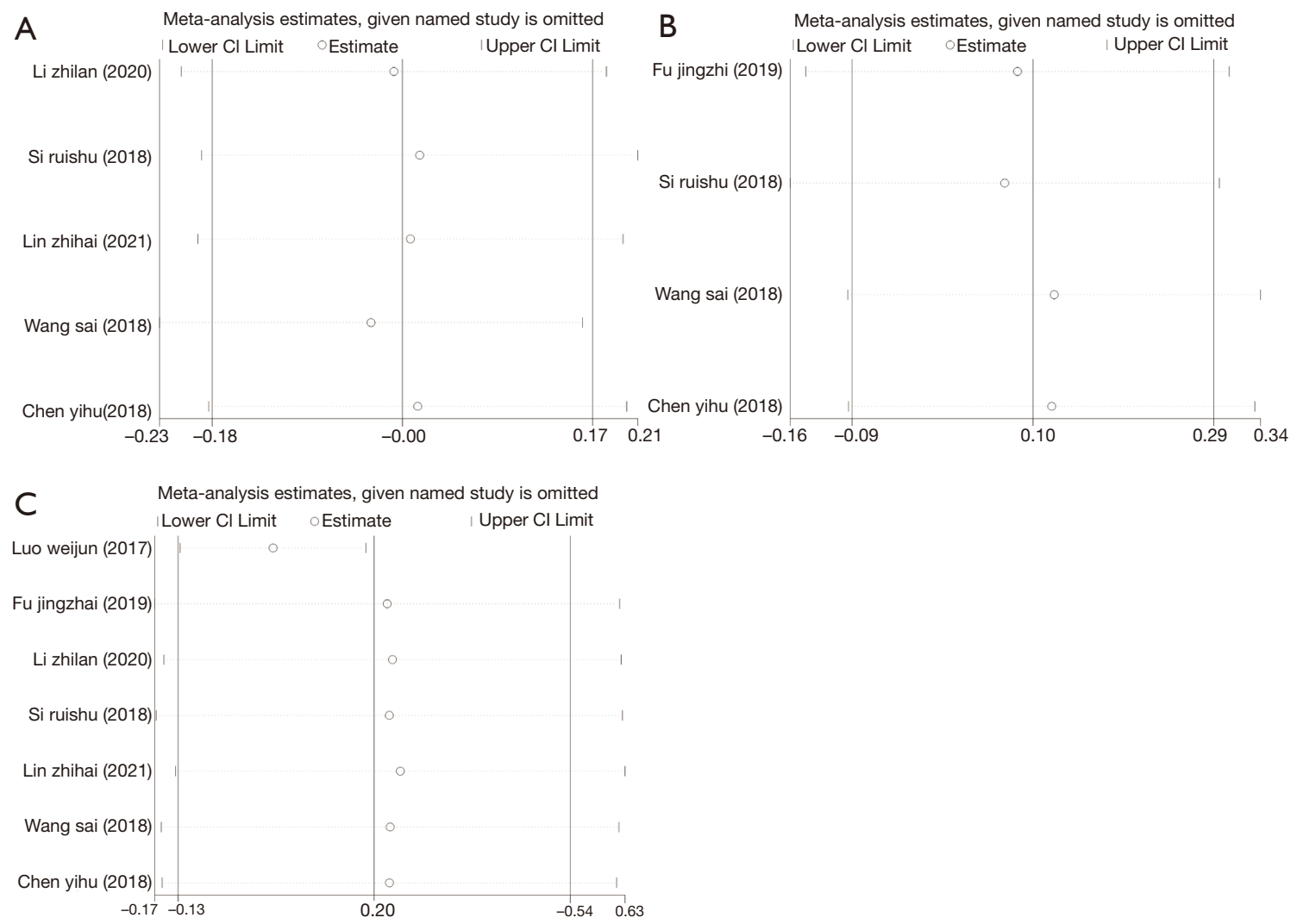

Figure 7 Sensitivity analysis of plot C-reactive protein (A), interleukin-6 (B), and tumor necrosis factor- $\alpha$ (C) levels before treatment. CI, confidence interval.

According to several studies (14-17,19-21), CRP level in the treatment group varied from $11.26-12.85 \mathrm{mg} / \mathrm{L}$ before treatment and decreased to $7.25-12.75 \mathrm{mg} / \mathrm{L}$ after treatment, while CRP level in the control group decreased from $5.80-13.12$ to $8.68-10.40 \mathrm{mg} / \mathrm{L}$. According to the literature analyzed $(15,17,20,21)$, the TNF- $\alpha$ level in the treatment group varied from $0.24-2.35 \mu \mathrm{g} / \mathrm{L}$ before treatment and decreased to $0.14-1.86 \mu \mathrm{g} / \mathrm{L}$ after treatment, while the TNF- $\alpha$ level in the control group decreased from $0.23-2.37$ to $0.17-2.37 \mu \mathrm{g} / \mathrm{L}$. The results show that the decrease of CRP and TNF- $\alpha$ levels in the control group was less than that in the treatment group.

Some studies have shown that inflammatory factors function in various stages of vasculopathy in CHD patients, including the formation and development of atherosclerosis, unstable plaque rupture, and thrombosis $(28,29)$. Aspirin can inhibit the formation of vascular inflammatory factors (CRP, TNF- $\alpha$ ), thus reducing vascular inflammation caused by atherosclerosis and ultimately protecting the integrity and normal function of vascular endothelium (7).

This meta-analysis has the following strengths. First, the types of studies included were all RCTs, representing the highest level of evidence quality for determining the effect of health care interventions. Second, a comprehensive, effective search strategy was used to screen literature, including pre-established screening criteria that reduced the bias. Third, data extraction and quality evaluation were completed independently by 2 investigators to minimize potential bias.

Our study also has some limitations. First, we did not have access to data from the original trial, so our information and judgment were limited by the analyzed literature's statistical analysis and outcome judgment criteria. Second, although the data on drug efficacy of aspirin are generally reported, there is a lack of comparison with other common drugs in clinical practice, such as metoprolol, nitrates, or nifedipine. Third, data on confounding factors are not available, so we cannot comment on the potential 

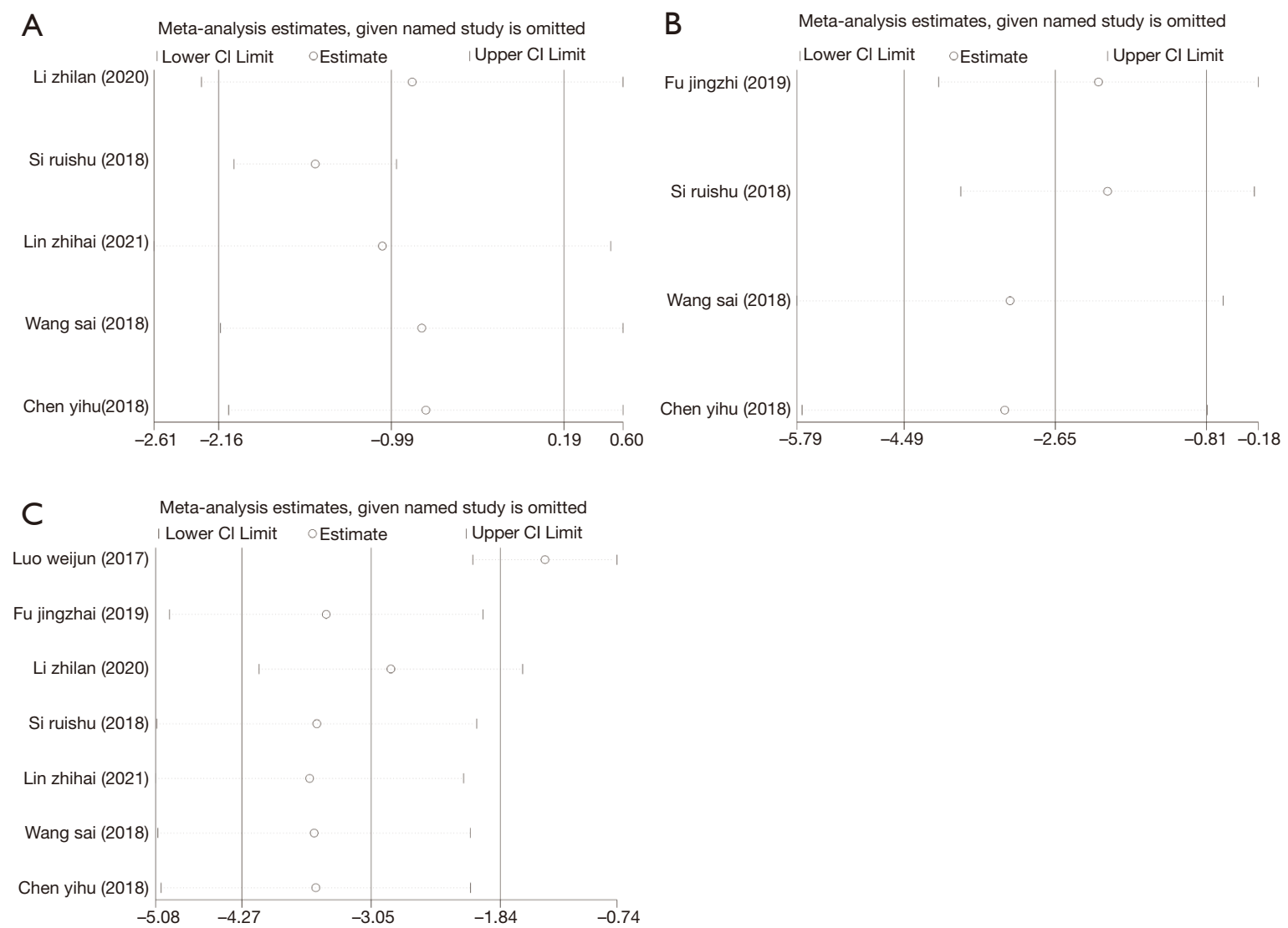

Figure 8 Sensitivity analysis of plot C-reactive protein (A), interleukin-6 (B), and tumor necrosis factor- (C) levels after treatment. CI, confidence interval.

effect of these on our meta-analysis results.

\section{Conclusions}

Aspirin is effective in the treatment of CHD, both alone and in combination. However, the latter has higher clinical efficacy and safety, can significantly reduce the level of inflammatory factors in CHD patients, and therefore can contribute to improvement in patient outcomes.

\section{Acknowledgments}

Funding: This study was support by a grant from the Basic Public Welfare Research Project of Zhejiang Province (no. LGJ18H020001).

\section{Footnote}

Reporting Checklist: The authors have completed the
PRISMA reporting checklist. Available at https://dx.doi. org/10.21037/apm-21-1648

Conflicts of Interest: All authors have completed the ICMJE uniform disclosure form (available at https://dx.doi. org/10.21037/apm-21-1648). The authors have no conflicts of interest to declare.

Ethical Statement: The authors are accountable for all aspects of the work in ensuring that questions related to the accuracy or integrity of any part of the work are appropriately investigated and resolved.

Open Access Statement: This is an Open Access article distributed in accordance with the Creative Commons Attribution-NonCommercial-NoDerivs 4.0 International License (CC BY-NC-ND 4.0), which permits the noncommercial replication and distribution of the article with the strict proviso that no changes or edits are made and the 
original work is properly cited (including links to both the formal publication through the relevant DOI and the license). See: https://creativecommons.org/licenses/by-nc-nd/4.0/.

\section{References}

1. De Hert M, Detraux J, Vancampfort D. The intriguing relationship between coronary heart disease and mental disorders. Dialogues Clin Neurosci 2018;20:31-40.

2. Expert Committee on Rational Drug Use of National Health and Family Planning Commission, Chinese Pharmacist Association. Guidelines for Rational Drug Use of Coronary Heart Disease (2nd Edition). Chinese Journal of the Frontiers of Medical Science (Electronic Version) 2018;10:1-130.

3. Nasiłowska-Barud A, Zapolski T, Barud M, et al. Overt and Covert Anxiety as a Toxic Factor in Ischemic Heart Disease in Women: The Link Between Psychological Factors and Heart Disease. Med Sci Monit 2017;23:751-8.

4. Joint Task Force for Guideline on the Assessment and Management of Cardiovascular Risk in China. Guideline on the assessment and management of cardiovascular risk in China. Zhonghua Yu Fang Yi Xue Za Zhi 2019;53:13-35.

5. Liu J. Progress in inflammatory pathogenesis of atherosclerosis. Journal of Xi' an Jiaotong University (Medical Sciences) 2015;36:141-52.

6. Wang Z. Interpretation of ACC/AHA Guideline for Primary Prevention of Cardiovascular Diseases in 2019. Chinese Circulation Journal 2019;34:82-5.

7. Hybiak J, Broniarek I, Kiryczyński G, et al. Aspirin and its pleiotropic application. Eur J Pharmacol 2020;866:172762.

8. Antithrombotic Trialists' (ATT) Collaboration; Baigent C, Blackwell L, et al. Aspirin in the primary and secondary prevention of vascular disease: collaborative meta-analysis of individual participant data from randomised trials. Lancet 2009;373:1849-60.

9. Santilli F, Rocca B, De Cristofaro R, et al. Platelet cyclooxygenase inhibition by low-dose aspirin is not reflected consistently by platelet function assays: implications for aspirin "resistance". J Am Coll Cardiol 2009;53:667-77.

10. Connolly SJ, Eikelboom JW, Bosch J, et al. Rivaroxaban with or without aspirin in patients with stable coronary artery disease: an international, randomised, double-blind, placebo-controlled trial. Lancet 2018;391:205-18.

11. Eikelboom JW, Connolly SJ, Bosch J, et al. Rivaroxaban with or without Aspirin in Stable Cardiovascular Disease.
N Engl J Med 2017;377:1319-30.

12. Anand SS, Bosch J, Eikelboom JW, et al. Rivaroxaban with or without aspirin in patients with stable peripheral or carotid artery disease: an international, randomised, double-blind, placebo-controlled trial. Lancet 2018;391:219-29.

13. Jin Y. Efficacy of Aspirin Enteric -coated Tablets Combined with Clopidogrel Bisulfate Tablets in the Treatment of Coronary Heart Disease and Angina Pectoris. China Foreign Medical Treatment 2020;39:100-2.

14. Luo W, Wu X, Li F, et al. Clinical effect of aspirin entericcoated tablets combined with clopidogrel on patients with coronary heart disease angina pectoris. China Modern Doctor 2017;55:122-4,127.

15. Fu J, Wang Y, Wang C, et al. Effectiveness of Aspirin Combined with Atorvastatin in Adjuvant Treatment of Patients with Coronary Athero-sclerotic Heart Disease and Its Effects on Blood Lipids, Inflammatory Factors and Quality of Life. Medical \& Pharmaceutical Journal of Chinese People's Liberation Army 2019;233:86-9.

16. Li Z, Li C. Study on the efficacy of aspirin combined with clopidogrel in the treatment of coronary heart disease in the elderly. China Practical Medical 2020;15:18-20.

17. Si R. Effects of the combination of atorvastatin and aspirin on blood lipids and inflammation cytokines in patients with coronary heart disease. Sichuan Journal of Physiological Sciences 2018;40:109-111.

18. Lin $Z$, Wang $Z, W u Z$, et al. Efficacy of betaloc combined with aspirin in patients with coronary heart disease and angina pectoris and its effect on serum hs-CRP, sCD40L and IL-6 levels. Journal of Clinical and Experimental Medicine 2021;20:259-62.

19. Huang H, Sun C. Comparative analysis of clinical efficacy and adverse reactions of clopidogrel and aspirin in the treatment of coronary heart disease. Chinese Journal of Modern Drug Application 2016;10:92-4.

20. Wang $S$. The effect of clopidogrel combined with aspirin on serum inflammatory factors and cardiac function in elderly patients with unstable angina pectoris. Chinese Journal of Gerontology 2018;38:529-31.

21. Chen Y. Effect of clopidogrel combined with aspirin on angina, serum inflammatory factors and cardiac function in elderly patients with coronary heart disease. The Journal of Medical Theory and Practice 2018;031:3198-200.

22. Liu Z. Efficacy of clopidogrel combined with aspirin in the treatment of elderly coronary heart disease in community. Chinese Community Doctors 2020;801:76-8. 
23. Zheng W. Value of Clopidogrel and Aspirin in the Treatment of Elderly Patients with Coronary Heart Disease. China Foreign Medical Treatment 2020;39:10-2.

24. Lei M. Investigation on the effect of clopidogrel and aspirin on reducing adverse reactions and improving the safety of treatment in patients with coronary heart disease. Xinxueguanbing Fangzhi Zhishi 2020;10:18-20.

25. Qiu X. Comparative study of clopidogrel and aspirin in the treatment of elderly patients with coronary heart disease. Journal of Clinical Medical Literature (Electronic Edition) 2020;7:161,164.

26. Grines CL, Bonow RO, Casey DE Jr, et al. Prevention of premature discontinuation of dual antiplatelet therapy in patients with coronary artery stents: a science advisory from the American Heart Association, American College of Cardiology, Society for Cardiovascular Angiography and Interventions, American College of Surgeons, and American Dental Association, with representation from the American College of Physicians. Catheter Cardiovasc Interv 2007;69:334-40.

27. Karmali KN, Lloyd-Jones DM, Berendsen MA, et al. Drugs for Primary Prevention of Atherosclerotic Cardiovascular Disease: An Overview of Systematic Reviews. JAMA Cardiol 2016;1:341-9.

28. Liao JK. Beyond lipid lowering: the role of statins in vascular protection. Int J Cardiol 2002;86:5-18.

29. Libby P. Changing concepts of atherogenesis. J Intern Med 2000;247:349-58.

(English Language Editors: M. Bucci and J. Gray)

Cite this article as: Mao P, Liu X, Weng Y, Tang L, Tang Y. Clinical efficacy of aspirin combination treatment in the treatment of coronary heart disease and its effect on inflammatory factors: a systematic review and meta-analysis. Ann Palliat Med 2021;10(8):8858-8868. doi: 10.21037/apm-211648 